\title{
CONTRA-SOMEWHAT CONTINUOUS FUNCTIONS
}

\author{
C. W. BAKER
}

\begin{abstract}
Two new forms of contra-somewhat continuity are introduced. Characterizations and the basic properties of both forms investigated.
\end{abstract}

\section{Introduction}

Gentry and Hoyle [8] introduced the class of somewhat continuous functions in 1971. These functions, which are a generalization of continuity requiring nonempty inverse images of open sets to have nonempty interiors instead of being open, have proved to be very useful in topology. Several variations of these functions have been studied. For example, Caldas, et al. [1] developed the notions of a somewhat $\beta$-continuous function and a somewhat $\beta$-open function in 2011. The concept of a contra-continuous function, which is a modification of continuity requiring inverse images of open sets to be closed rather than open, was introduced by Dontchev [3] in 1996. The purpose of this note is to combine these two concepts. There are two obvious ways to form contra versions of somewhat continuity. Both forms, which we call contra-1-somewhat continuity and contra-2-somewhat continuity, are investigated in this note. Characterizations and properties of both forms are developed. It is shown that the two forms are independent. Also relationships between these forms and various types of contra-continuity are determined. Contra-continuity implies both forms of contra-somewhat continuity and contra-semicontinuity implies contra-1-somewhat continuity. Both forms are characterized by their actions on dense sets and sets with large kernels. Specifically, contra-1-somewhat continuity is characterized by requiring images of dense sets to have maximum kernels and contra2 -somewhat continuity is characterized by requiring images of sets with maximum kernels to be dense in the range. Relationships between these classes of functions and the concept of equivalent topologies are examined.

\section{Preliminaries}

The symbols $X$ and $Y$ represent topological spaces with no separation properties assumed unless explicitly stated. All sets are considered to be 


\section{W. BAKER}

subsets of topological spaces. The closure and interior of a set $A$ are signified by $\operatorname{Cl}(A)$ and $\operatorname{Int}(A)$, respectively. A set $A$ is said to be preopen [12] (respectively, semiopen [10]) if $A \subseteq \operatorname{Int}(\mathrm{Cl}(A)$ ), (respectively, $A \subseteq \mathrm{Cl}(\operatorname{Int}(A)))$. A set $A$ is preclosed [12] (respectively, semi-closed [2]) provided its complement is preopen (respectively, semi-open). The intersection of all semi-closed sets containing $A$ is called the semi-closure of $A$ [2] and denoted by $\operatorname{sCl}(A)$. A set $A$ is regular open (respectively, regular closed) if $\operatorname{Int}(\mathrm{Cl}(A))=A$ (respectively, $\mathrm{Cl}(\operatorname{Int}(A))=A)$. A set $A$ is called $\delta$-open [16] if for each $x \in A$ there exists a regular open set $U$ such that $x \in U \subseteq A$ and the set $\{x \in A: x \in U \subseteq A$ for some regular open set $U\}$ is called the $\delta$-interior of $A$ and is denoted by $\delta$ - $\operatorname{Int}(A)$. A set $A$ is called $\delta$-semiopen [14] if $A \subseteq \mathrm{Cl}(\delta$ - $\operatorname{Int}(A))$.

Definition 1. A function $f: X \rightarrow Y$ is said to be somewhat continuous [8] if for every open subset $V$ of $Y$ such that $f^{-1}(V) \neq \emptyset$ there exists an open subset $U$ of $X$ such that $U \neq \emptyset$ and $U \subseteq f^{-1}(V)$.

Definition 2. [7] A function $f: X \rightarrow Y$ is said to be $\delta$-semicontinuous at a point $x \in X$ if for each open set $V$ in $Y$ containing $f(x)$, there exists a $\delta$-semiopen set $U$ in $X$ containing $x$ such that $f(U) \subseteq V$, and $f$ is said to be $\delta$-semicontinuous if it has this property at each point of $X$.

Definition 3. A function $f: X \rightarrow Y$ is said to be contra-continuous [3] if $f^{-1}(V)$ is closed for every open subset $V$ of $Y$.

Definition 4. A function $f: X \rightarrow Y$ is said to be contra-precontinuous [9] (respectively, contra-semicontinuous [4]) if $f^{-1}(V)$ is preclosed (respectively, semi-closed) for every open subset $V$ of $Y$.

Definition 5. [6] A function $f: X \rightarrow Y$ is said to be contra $\delta$-semicontinuous at a point $x \in X$ if for each closed set $F$ in $Y$ with $f(x) \in F$, there exists a $\delta$-semiopen set $U$ in $X$ such that $x \in U$ and $f(U) \subseteq F$, and $f$ is called contra $\delta$-semicontinuous if it has this property at each point of $X$.

Definition 6. A subset $A$ of a space $X$ is said to be feebly open [11] if there exists an open set $U$ in $X$ such that $U \subseteq A \subseteq s C l(U)$.

Definition 7. [5] A function $f: X \rightarrow Y$ is said to be $\delta_{s}$-na-continuous at a point $x \in X$ if for each feebly open set $V$ in $Y$ containing $f(x)$, there exists a $\delta$-semiopen set $U$ in $X$ containing $x$ such that $f(U) \subseteq V$, and $f$ is said to be $\delta_{s}$-na-continuous if it has this property at each point of $X$.

Definition 8. Let $A$ be a subset of a space $X$. The kernel of $A$ [13], denoted by $\operatorname{ker}(A)$, is the intersection of all open subsets of $X$ containing $A$.

Lemma 2.1. [9] The following statements hold for subsets $A$ and $B$ of a space $X$ : 


\section{CONTRA-SOMEWHAT CONTINUOUS FUNCTIONS}

(a) $x \in \operatorname{ker}(A)$ if and only if $A \cap F \neq \emptyset$ for every closed subset $F$ of $X$ containing $x$.

(b) $A \subseteq \operatorname{ker}(A)$ and $A=\operatorname{ker}(A)$ if $A$ is open in $X$.

(c) If $A \subseteq B$, then $\operatorname{ker}(A) \subseteq \operatorname{ker}(B)$.

Lemma 2.2. If $A$ is a subset of a space $X$, then $\operatorname{ker}(\operatorname{ker}(A))=\operatorname{ker}(A)$.

Proof. Since by Lemma 2.1 (b) $A \subseteq \operatorname{ker}(A)$, it follows from Lemma 2.1 (c) that $\operatorname{ker}(A) \subseteq \operatorname{ker}(\operatorname{ker}(A))$. To see that the reverse inclusion holds, let $V$ be an open set such that $A \subseteq V$. By definition $\operatorname{ker}(A) \subseteq V$. Then using Lemma 2.1 (c) and (b) we have $\operatorname{ker}(\operatorname{ker}(A)) \subseteq \operatorname{ker}(V)=V$. It then follows that $\operatorname{ker}(\operatorname{ker}(A)) \subseteq \operatorname{ker}(A)$.

Lemma 2.3. Let $A$ and $B$ be subsets of a space $X$ such that $A \subseteq B$. Then $\operatorname{ker}(A)=\operatorname{ker}(B)$ if and only if $\operatorname{ker}_{B}(A)=B$.

Proof. $(\Rightarrow)$ Assume $\operatorname{ker}(A)=\operatorname{ker}(B)$. Obviously $\operatorname{ker}_{B}(A) \subseteq B$. Let $x \in B$. Then $x \in \operatorname{ker}(B)$ and hence, $x \in \operatorname{ker}(A)$. Therefore for every open set $V$ containing $A$, we have $x \in V \cap B$. Thus, $x \in \operatorname{ker}_{B}(A)$. Hence, we have $\operatorname{ker}_{B}(A)=B$.

$(\Leftarrow)$ Assume $\operatorname{ker}_{B}(A)=B$. Then $B=\cap\{V \cap B: V$ is open in $X$ and $A \subseteq$ $V\} \subseteq \cap\{V: V$ is open in $X$ and $A \subseteq V\}=\operatorname{ker}(A)$. Thus, $B \subseteq \operatorname{ker}(A)$ and hence, $\operatorname{ker}(B) \subseteq \operatorname{ker}(\operatorname{ker}(A))=\operatorname{ker}(A)$. Finally, since $A \subseteq B$, $\operatorname{ker}(A) \subseteq$ $\operatorname{ker}(B)$ and hence, $\operatorname{ker}(A)=\operatorname{ker}(B)$.

\section{Contra-1-Somewhat Continuous Functions}

Definition 9. A function $f: X \rightarrow Y$ is said to be contra-1-somewhat continuous provided that for every closed set $F \subseteq Y$ such that $f^{-1}(F) \neq \emptyset$, there exists an open set $U \subseteq X$ such that $\emptyset \neq U \subseteq f^{-1}(F)$.

Theorem 3.1. For a function $f: X \rightarrow Y$ the following conditions are equivalent:

(a) $f$ is contra-1-somewhat continuous.

(b) For every open set $V \subseteq Y$ such that $f^{-1}(V) \neq X$, there exists a closed set $F \subseteq X$ such that $F \neq X$ and $f^{-1}(V) \subseteq F$.

(c) For every dense set $M \subseteq X, \operatorname{ker}(f(M))=\operatorname{ker}(f(X))$.

Proof. (a) $\Rightarrow$ (b). Let $V$ be an open subset of $Y$ such that $f^{-1}(V) \neq X$. Then, since $f^{-1}(Y-V) \neq \emptyset$, there exists on open set $U \subseteq X$ for which $\emptyset \neq U \subseteq f^{-1}(Y-V)$ and hence, $f^{-1}(V) \subseteq X-U$. Since $U \neq \emptyset, X-U \neq X$ and thus, $X-U$ is the desired set.

(b) $\Rightarrow$ (c). Let $M$ be a dense subset of $X$. Let $V$ be an open subset of $X$. Suppose $f(X) \nsubseteq V$. Then $X \nsubseteq f^{-1}(V)$ and by (b) there exists a closed set $F \subseteq X$ such that $F \neq X$ and $f^{-1}(V) \subseteq F$. Since $M$ is dense, $M \not \subset F$ and hence, $M \nsubseteq f^{-1}(V)$ and $f(M) \nsubseteq V$. It then follows that $\operatorname{ker}(f(X)) \subseteq$ 


\section{W. BAKER}

$\operatorname{ker}(f(M))$. By Lemma 2.1, since $M \subseteq X, \operatorname{ker}(f(M)) \subseteq \operatorname{ker}(f(X))$ and thus, $\operatorname{ker}(f(M))=\operatorname{ker}(f(X))$.

(c) $\Rightarrow($ a). Suppose $f$ is not contra-1-somewhat continuous. Then there exists $F \subseteq Y$ such that $F$ is closed, $f^{-1}(F) \neq \emptyset$, and for every $U \subseteq X$ such that $U$ is open and $U \neq \emptyset, U \nsubseteq f^{-1}(F)$. Then for every nonempty open subset $U$ of $X, U \cap\left(X-f^{-1}(F)\right) \neq \emptyset$. Thus $X-f^{-1}(F)$ is dense in $X$. Therefore using (c) we obtain $f(X) \subseteq \operatorname{ker}(f(X))=\operatorname{ker}\left(f\left(X-f^{-1}(F)\right)\right) \subseteq$ $\operatorname{ker}(Y-F)=Y-F$. Thus, $f(X) \subseteq Y-F$ and hence, $f^{-1}(F)=\emptyset$. This contradiction proves that $f$ is contra-1-somewhat continuous.

The next result follows from Lemma 2.3.

Corollary 3.2. A function $f: X \rightarrow Y$ is contra-1-somewhat continuous if and only if for every dense subset $M$ of $X \operatorname{ker}_{f(X)}(f(M))=f(X)$.

Corollary 3.3. If $f: X \rightarrow Y$ is contra-1-somewhat continuous and surjective, then for every dense subset $M$ of $X, \operatorname{ker}(f(M))=Y$.

Corollary 3.4. If $f: X \rightarrow Y$ is contra-1-somewhat continuous and $f(X)$ is open in $Y$, then for every dense subset $M$ of $X, \operatorname{ker}(f(M))=f(X)$.

Theorem 3.5. If $f: X \rightarrow Y$ is contra-semicontinuous, then $f$ is contra1-somewhat continuous.

Proof. Let $F \subseteq Y$ such that $F$ is closed and $f^{-1}(F) \neq \emptyset$. Since $f^{-1}(F)$ is semi-open, $f^{-1}(F) \subseteq \mathrm{Cl}\left(\operatorname{Int}\left(f^{-1}(F)\right)\right)$. Then $\operatorname{Int}\left(f^{-1}(F)\right) \neq \emptyset$ and hence, $f$ is contra-1-somewhat continuous.

Corollary 3.6. If $f: X \rightarrow Y$ is contra $\delta$-semicontinuous, then $f$ is contra1-somewhat continuous.

Theorem 3.7. If $f: X \rightarrow Y$ is semicontinuous, and $X$ has no proper open dense set, then $f$ is contra-1-somewhat continuous.

Proof. Let $V \subseteq Y$ be an open set such that $f^{-1}(V) \neq X$. Then $f^{-1}(V)$ is semiopen and hence, $f^{-1}(V) \subseteq \mathrm{Cl}\left(\operatorname{Int}\left(f^{-1}(V)\right)\right)$. Since $\operatorname{Int}\left(f^{-1}(V)\right)$ is open, it is not dense in $X$ and therefore, $\operatorname{Cl}\left(\operatorname{Int}\left(f^{-1}(V)\right)\right) \neq X$. Thus, by Theorem 3.1 (b) $f$ is contra-1-somewhat continuous.

Corollary 3.8. If $f: X \rightarrow Y$ is $\delta$-semicontinuous, and $X$ has no proper open dense set, then $f$ is contra-1-somewhat continuous.

Corollary 3.9. If $f: X \rightarrow Y$ is $\delta_{s}$-na-continuous, and $X$ has no proper open dense set, then $f$ is contra-1-somewhat continuous.

Theorem 3.10. If $f: X \rightarrow Y$ is contra-1-somewhat continuous, and $A$ is a dense subset of $X$, then $\left.f\right|_{A}: A \rightarrow Y$ is contra-1-somewhat continuous. 


\section{CONTRA-SOMEWHAT CONTINUOUS FUNCTIONS}

Proof. Let $F \subseteq Y$ be a closed set such that $\left.f\right|_{A} ^{-1}(F) \neq \emptyset$. Then $f^{-1}(F) \neq \emptyset$ and, since $f$ is contra-1-somewhat continuous, there exists an open set $U \subseteq X$ such that $\emptyset \neq U \subseteq f^{-1}(F)$. Then $\left.U \cap A \subseteq f\right|_{A} ^{-1}(F)$ and, since $A$ is dense in $X, U \cap A \neq \emptyset$. Thus, $\left.f\right|_{A}: A \rightarrow Y$ is contra-1-somewhat continuous.

The following example shows that the requirement that $A$ be dense is necessary.

Example 3.11. Let $X=\{a, b, c\}$ have the topologies $\tau=\{X, \emptyset,\{a\}\}$ and $\sigma=\{X, \emptyset,\{c\}\}$. Then the identity mapping $f:(X, \tau) \rightarrow(X, \sigma)$ is contra1-somewhat continuous, but, if $A=\{b, c\}$ and $\tau_{A}$ is the relative topology on $A$, then $\left.f\right|_{A}:\left(A, \tau_{A}\right) \rightarrow(X, \sigma)$ is not contra-1-somewhat continuous.

Theorem 3.12. If $A$ is an open dense subset of a space $X$ and $f: A \rightarrow Y$ is a contra-1-somewhat continuous function such that $\operatorname{ker}(f(A))=Y$, then any extension of $f, g: X \rightarrow Y$, is contra-1-somewhat continuous.

Proof. Let $F \subseteq Y$ be a closed set such that $g^{-1}(F) \neq \emptyset$. Since $\operatorname{ker}(f(A))=$ $Y, f(A) \nsubseteq Y Y-F$ and hence, $f(A) \cap F \neq \emptyset$. Thus, $f^{-1}(F) \neq \emptyset$. So there exists $U \subseteq A$ such that $U$ is open in $A$ and $\emptyset \neq U \subseteq f^{-1}(F) \subseteq g^{-1}(F)$. Finally, since $A$ is open in $X, U$ is open in $X$ and hence, $g: X \rightarrow Y$ is contra-1-somewhat continuous.

The proof of the following result is straightforward.

Theorem 3.13. If $X=A \cup B$, where $A$ and $B$ are open sets and $f: X \rightarrow Y$ is a function such that $\left.f\right|_{A}: A \rightarrow Y$ and $\left.f\right|_{B}: B \rightarrow Y$ are contra-1somewhat continuous, then $f$ is contra-1-somewhat continuous.

Definition 10. Let $X$ be a set. Topologies $\tau$ and $\tau^{\prime}$ on $X$ are said to be weakly equivalent [8] provided that, if $U \in \tau$ and $U \neq \emptyset$, then there exists $V \in \tau^{\prime}$ such that $V \subseteq U$ and $V \neq \emptyset$ and, if $U \in \tau^{\prime}$ and $U \neq \emptyset$, then there exists $V \in \tau$ such that $V \subseteq U$ and $V \neq \emptyset$.

The proof of the following theorem is straightforward and is omitted.

Theorem 3.14. If $f:(X, \tau) \rightarrow(Y, \sigma)$ is contra-1-somewhat continuous and $\tau^{\prime}$ is a topology on $X$ that is weakly equivalent to $\tau$, then $f:\left(X, \tau^{\prime}\right) \rightarrow$ $(Y, \sigma)$ is contra-1-somewhat continuous.

Definition 11. Let $X$ be a set. Topologies $\tau$ and $\tau^{\prime}$ on $X$ are said to be contra weakly equivalent provided that, if $F$ is $\tau$-closed and $F \neq \emptyset$, then there exists a $\tau^{\prime}$-closed set $G$ such that $G \subseteq F$ and $G \neq \emptyset$ and, if $F$ is a $\tau^{\prime}$-closed set and $F \neq \emptyset$, then there exists a $\bar{\tau}$-closed set $G$ such that $G \subseteq U$ and $G \neq \emptyset$. 


\section{W. BAKER}

Theorem 3.15. If $f:(X, \tau) \rightarrow(Y, \sigma)$ is contra-1-somewhat continuous and surjective and $\sigma^{\prime}$ is a topology on $Y$ that is contra weakly equivalent to $\sigma$, then $f:(X, \tau) \rightarrow\left(Y, \sigma^{\prime}\right)$ is contra-1-somewhat continuous.

Proof. Let $F$ be a $\sigma^{\prime}$-closed subset of $Y$ such that $f^{-1}(F) \neq \emptyset$. Since $\sigma^{\prime}$ is contra-weakly equivalent to $\sigma$, there exists a nonempty $\sigma$-closed subset $G$ of $Y$ such that $G \subseteq F$. Since $f$ is surjective, $f^{-1}(G) \neq \emptyset$. So there exists $V \in \tau$ such that $V \neq \emptyset$ and $V \subseteq f^{-1}(G)$. Thus, $V \subseteq f^{-1}(F)$, which proves that $f:(X, \tau) \rightarrow\left(Y, \sigma^{\prime}\right)$ is contra-1-somewhat continuous.

Example 3.16. Let $X$ denote the real numbers and let $\tau$ be the usual topology on $X$ and $\tau^{\prime}$ the discrete topology on $X$. Since both $(X, \tau)$ and $\left(X, \tau^{\prime}\right)$ are $T_{1}, \tau$ and $\tau^{\prime}$ are contra-weakly equivalent, However they are not weakly equivalent.

\section{Contra-2-Somewhat Continuous Functions}

Definition 12. A function $f: X \rightarrow Y$ is said to be contra-2-somewhat continuous if for every open set $V \subset Y$ such that $f^{-1}(V) \neq \emptyset$, there exists a closed set $F \subseteq X$ such that $\emptyset \neq F \subseteq f^{-1}(V)$.

Theorem 4.1. For a function $f: X \rightarrow Y$ the following conditions are equivalent:

(a) $f$ is contra-2-somewhat continuous.

(b) For every closed set $F \subseteq Y$ such that $f^{-1}(F) \neq X$, there exists an open set $U \subseteq X$ such that $U \neq X$ and $f^{-1}(F) \subseteq U$.

(c) For every set $A \subseteq X$, such that $\operatorname{ker}(A)=X, f(A)$ is dense in $f(X)$.

The proof is analogous to that of Theorem 3.1.

The function in Example 3.11 is contra-1-somewhat continuous but not contra-2-somewhat continuous. The following example completes the proof that these two concepts are independent.

Example 4.2. Let $X=\{a, b, c\}$ have the topologies $\tau=\{X, \emptyset,\{b, c\}\}$ and $\sigma=\{X, \emptyset,\{a, b\}\}$. Then the identity mapping $f:(X, \tau) \rightarrow(X, \sigma)$ is contra-2-somewhat continuous, but not contra-1-somewhat continuous.

The proof of the following result is straightforward.

Theorem 4.3. If $f: X \rightarrow Y$ is contra-continuous, then $f$ is contra-2somewhat continuous.

Since every function with a $T_{1}$-domain is contra-2-somewhat continuous, obviously contra-2-somewhat continuity does not imply contra-continuity.

Theorem 4.4. If $f: X \rightarrow Y$ is contra-precontinuous and somewhat continuous, then $f$ is contra-2-somewhat continuous. 


\section{CONTRA-SOMEWHAT CONTINUOUS FUNCTIONS}

Proof. Let $V \subseteq Y$ such that $V$ is open and $f^{-1}(V) \neq \emptyset$. Since $f$ is somewhat continuous, $\operatorname{Int}\left(f^{-1}(V)\right) \neq \emptyset$. Since $f$ is contra-precontinuous, $f^{-1}(V)$ is preclosed and therefore, $\operatorname{Cl}\left(\operatorname{Int}\left(f^{-1}(V)\right)\right) \subseteq f^{-1}(V)$. Since $\operatorname{Int}\left(f^{-1}(V)\right) \neq \emptyset, \mathrm{Cl}\left(\operatorname{Int}\left(f^{-1}(V)\right)\right) \neq \emptyset$. Hence, $f$ is contra-2-somewhat continuous.

Example 4.5. If $X$ is the real numbers, $\tau$ is the usual topology on $X$, and $\sigma$ is the discrete topology on $X$, then the identity mapping $f:(X, \tau) \rightarrow$ $(Y, \sigma)$ is contra-2-somewhat continuous but neither contra-precontinuous nor somewhat continuous.

Theorem 4.6. If $f: X \rightarrow Y$ is contra-2-somewhat continuous, and $A \subseteq X$ such that $\operatorname{ker}(A)=X$, then $\left.f\right|_{A}: A \rightarrow Y$ is contra-2-somewhat continuous.

Proof. Let $V$ be an open subset of $Y$ such that $\left.f\right|_{A} ^{-1}(V) \neq \emptyset$. Then $f^{-1}(V) \neq \emptyset$ and hence there exists a closed set $F \subseteq X$ such that $\emptyset \neq$ $F \subseteq f^{-1}(V)$. Then $F \cap A \subseteq f^{-1}(V) \cap A=\left.f\right|_{A} ^{-1}(V)$. Since $\operatorname{ker}(A)=X$ and $F \neq \emptyset, A \nsubseteq X-F$. Thus, $F \cap A \neq \emptyset$. Therefore $F \cap A$ is a nonempty set that is closed in $A$ for which $\left.F \cap A \subseteq f\right|_{A} ^{-1}(V)$, which proves that $\left.f\right|_{A}: A \rightarrow Y$ is contra-2-somewhat continuous.

Theorem 4.7. Let $A$ be a closed subset of a space $X$ and let $f: A \rightarrow Y$ be a contra-2-somewhat continuous function such that $f(A)$ is dense in $Y$. If $g: X \rightarrow Y$ is an extension of $f$, then $g$ is contra-2-somewhat continuous.

Proof. Let $V \subseteq Y$ be an open set such that $g^{-1}(V) \neq \emptyset$. Since $f(A)$ is dense in $Y, f(A) \cap V \neq \emptyset$ and hence, $f^{-1}(V) \neq \emptyset$. Since $f$ is contra-2somewhat continuous, there exists $F \subseteq A$ such that $F$ is closed in $A$ and $\emptyset \neq F \subseteq f^{-1}(V) \subseteq g^{-1}(V)$. Since $A$ is closed in $X, F$ is closed in $X$. Thus, $g$ is contra-2-somewhat continuous.

The proof of the following result is straightforward.

Theorem 4.8. Let $X=A \cup B$, where $A$ and $B$ are closed sets. If $f$ : $X \rightarrow Y$ is a function such that $\left.f\right|_{A}: A \rightarrow Y$ and $\left.f\right|_{B}: B \rightarrow Y$ are contra-2-somewhat continuous, then $f$ is contra-2-somewhat continuous.

Definition 13. A space $X$ is said to be quasiregular [15] if for every nonempty open subset $V$ of $X$, there exists a nonempty open set $U$ such that $C l(U) \subseteq V$.

The proof of the next theorem follows immediately from the definitions.

Theorem 4.9. If $X$ is quasiregular and $f: X \rightarrow Y$ is somewhat continuous, then $f$ is contra-2-somewhat continuous.

The proofs of the following two results are analogous to those of Theorems 3.14 and 3.15 .

MISSOURI J. OF MATH. SCI., FALL 2015 


\section{W. BAKER}

Theorem 4.10. If $f:(X, \tau) \rightarrow(Y, \sigma)$ is contra-2-somewhat continuous and surjective and $\sigma^{\prime}$ is a topology on $Y$ that is weakly equivalent to $\sigma$, then $f:(X, \tau) \rightarrow\left(Y, \sigma^{\prime}\right)$ is contra-2-somewhat continuous.

Theorem 4.11. If $f:(X, \tau) \rightarrow(Y, \sigma)$ is contra-2-somewhat continuous and $\tau^{\prime}$ is a topology on $X$ that is contra weakly equivalent to $\tau$, then $f$ : $\left(X, \tau^{\prime}\right) \rightarrow(Y, \sigma)$ is contra-2-somewhat continuous.

\section{REFERENCES}

[1] M. Caldas, S. Jafari, and G. Navalai, On somewhat $\beta$-continuity, somewhat $\beta$ openness and hardly $\beta$-openness, Internat. J. Gen. Top., 4 (2011), 1-7.

[2] S. G. Crossley and S. K. Hildebrand, Semi-closure, Texas J. Sci., 22 (1971), 99-112.

[3] J. Dontchev, Contra-continuous functions and strongly S-closed spaces, doi: 10.1155/S0161171296000427, Internat. J. Math. Math. Sci., 19 (1996), 303-310.

[4] J. Dontchev and T. Noiri, Contra-semicontinuous functions, Mathematica Pannonica, 10 (1999), 159-168.

[5] E. Ekici, On an extension for functions, Demonstratio Mathematica, XXXIX.3 (2006), 657-670.

[6] E. Ekici, On a weaker form of RC-continuity, Analele Universităţii de Vest din Timişoara, Seria Matematică-Informatică, XLII, fasc., 1 (2004), 79-91.

[7] E. Ekici and G. Navalagi, $\delta$-semicontinuous functions, Mathematical Forum, XVII (2004-2005), 29-42.

[8] K. R. Gentry and H. B. Hoyle, III, Somewhat continuous functions, Czech. Math. J., 21 (1971), 5-12.

[9] S. Jafari and T. Noiri, On contra-precontinuous functions, Bull. Malaysian Math Sci. Soc., 25 (2002), 115-128.

[10] N. Levine, Semi-open sets and semi-continuity in topological spaces, Amer. Math. Monthly, 70 (1963), 36-41.

[11] S. N. Maheshwari and U. Tapi, Note on some applications on feebly open sets, M. B. J. Univ. Saugar, 1979

[12] A. S. Mashhour, M. E. Abd El-Monsef, and S. N. El-Deep, On precontinuous and weak precontinuous mappings, Proc. Math. Phys. Soc. Egypt, 53 (1982), 47-53.

[13] M. Mršević, On pairwise $R_{0}$ and pairwise $R_{1}$ bitopological spaces, Bull. Math. Soc. Sci. Math. R. S. Roumanie, 30 (1986), 141-148.

[14] J. H. Park, B. Y. Lee, and M. J. Son, On $\delta$-semiopen sets in topological spaces, J. Indian Acad. Math., 19.1 (1997), 59-67.

[15] J. R. Porter and R. G. Woods, Extensions and Absolutes of Hausdorff Spaces, Springer-Verlag, New York, 1988

[16] N. V. Veličko, H-closed topological spaces, Amer. Math. Soc. Transl., 78.2 (1968), $103-118$.

MSC2010: 54C08, 54C10

Key words and phrases: somewhat continuity, contra-continuity, contra-1somewhat continuity, contra-2-somewhat continuity

Department of Mathematics, Indiana University Southeast, New Albany, in 47150

E-mail address: cbaker@ius.edu 